This item was submitted to Loughborough's Research Repository by the author.

Items in Figshare are protected by copyright, with all rights reserved, unless otherwise indicated.

\title{
Design for sustainable behaviour: strategies and perceptions
}

PLEASE CITE THE PUBLISHED VERSION

http://dx.doi.org/10.1016/j.destud.2009.05.001

PUBLISHER

(c) Elsevier

VERSION

AM (Accepted Manuscript)

LICENCE

CC BY-NC-ND 4.0

REPOSITORY RECORD

Lilley, Debra. 2019. "Design for Sustainable Behaviour: Strategies and Perceptions". figshare. https://hdl.handle.net/2134/11817. 
This item was submitted to Loughborough's Institutional Repository (https://dspace.lboro.ac.uk/) by the author and is made available under the following Creative Commons Licence conditions.

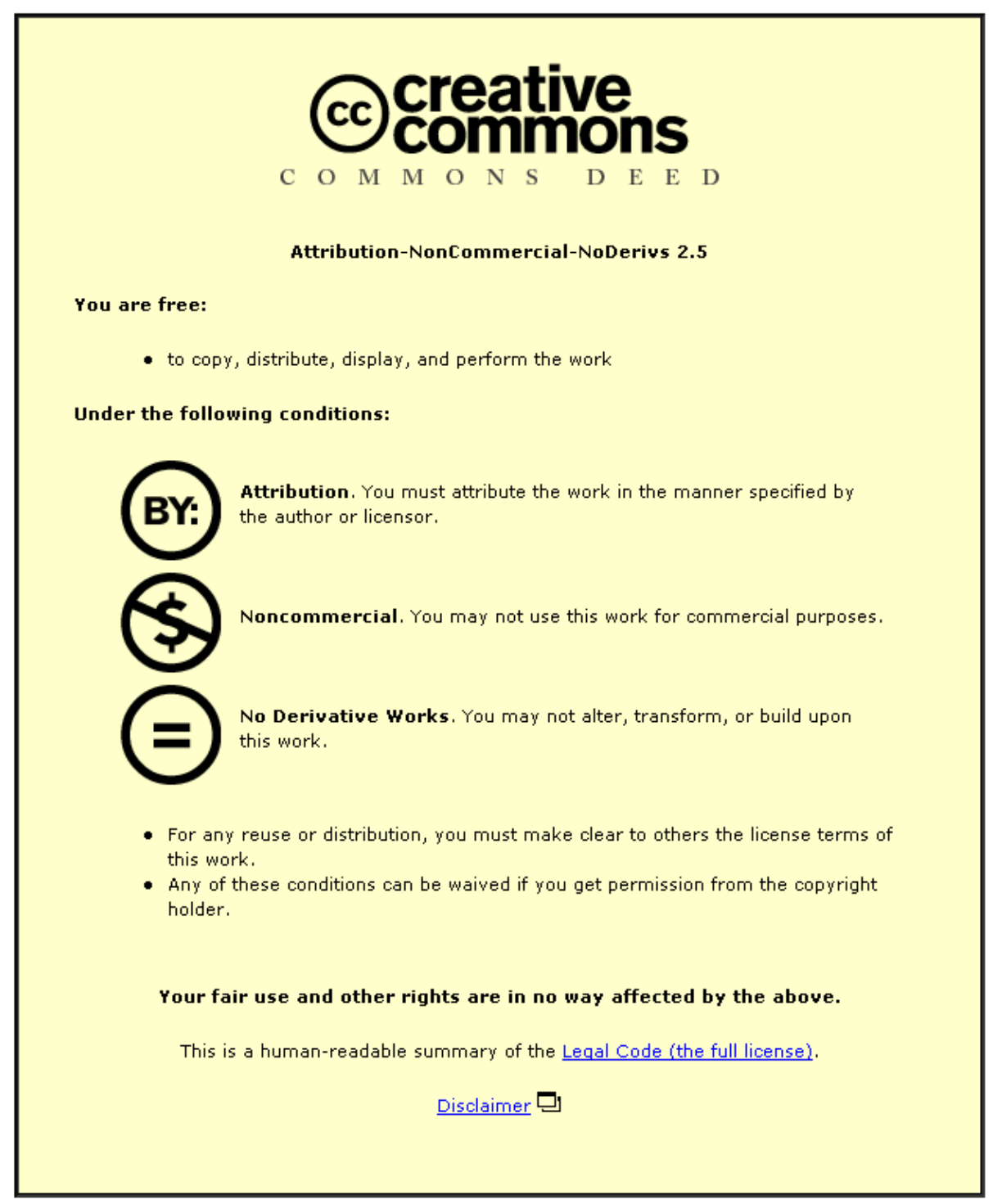

For the full text of this licence, please go to: http://creativecommons.org/licenses/by-nc-nd/2.5/ 


\title{
Design for Sustainable Behaviour: Strategies and Perceptions Dr Debra Lilley, Loughborough University
}

\begin{abstract}
This paper presents selected findings of doctoral research exploring how design could be used to influence user behaviour towards more sustainable practices. It describes three strategies for changing user behaviour through design drawn from literature and outlines the methodology and findings of a case study exploring the application of these strategies in sustainable design. Drawing on the perceptions of design professionals interviewed in response to one of the concepts generated, the paper goes on to explore the perceived acceptability and effectiveness of these strategies. It concludes by commenting on the wider implications of these perceptions for ongoing research.
\end{abstract}

\section{Keywords: user behaviour, environmental design, design techniques.}

Sustainable design takes into account environmental, economic and social impacts enacted throughout the product lifecycle (Bhamra and Lofthouse, 2007). These interrelated domains are often referred to as the three pillars or triple bottom line of sustainability (Elkington, 1997). Whereas economic and environmental concerns are generally well defined and understood, the social sphere of sustainable design is less so (Colantonio, 2007) and as such warrants further explanation. In its broadest terms it can encompass; personal responsibility, quality of life, health, well-being and happiness, democratic participation and cooperative behaviour (Colantonio, 2007, Baines and Morgan, 2004, Sinner et al., 2004, Polese and Stren, 2000).

Designers shape the development of products and services which directly impact upon society and the environment (Papanek, 1971). The application of sustainable design strategies can greatly reduce lifecycle impacts (Lewis et al., 2001). Impacts which occur during use, however, are often determined by consumer behaviour (Bhamra et al., 2008). Influencing user behaviour can be challenging. In spite of over a decade of campaigns exhorting consumers to behave differently and greater product efficiency, consumers are slow to adopt more sustainable behaviours (DeVries, 2006, Siegle, 2006) and behavioural changes made are often short-lived (Scott, 2004).

Designers are in a position to reduce use impacts by purposefully shaping behaviour towards more sustainable practices (Lockton et al., 2008, Elias et al., 2008, Bhamra et al., 2008, Wever et al., 2008). Early research identified Eco-feedback (McCalley and Midden, 2006), Behaviour Steering (Jelsma and Knot, 2002, Akrich, 1992) and Persuasive Technology or Captology (Fogg, 2003) as potential strategies which could be integrated into product design to influence user behaviour (Lilley. D). Figure 1 describes each approach and indicates the degree to which power in decision-making is retained by the user or delegated to the product.

Further research led to the classification of seven strategies, described in full in Bhamra et. al, 2008. Whilst providing interesting considerations for designers, these strategies have not been widely applied and there is lack of data on their effectiveness and acceptability. It is for this reason that empirical research was conducted.

\section{Methodology}

To generate documentary evidence of the process and results of the application of behaviour change strategies in sustainable design, primary research was required. To this end, a series of qualitative research studies were carried out within the framework of an overarching case study methodology which formed part of Lilley's doctoral research (Lilley, 2007). The case study explored the social impacts of mobile phone use in public and how the application of one or more of the strategies described in Figure 1 could reduce these impacts. 


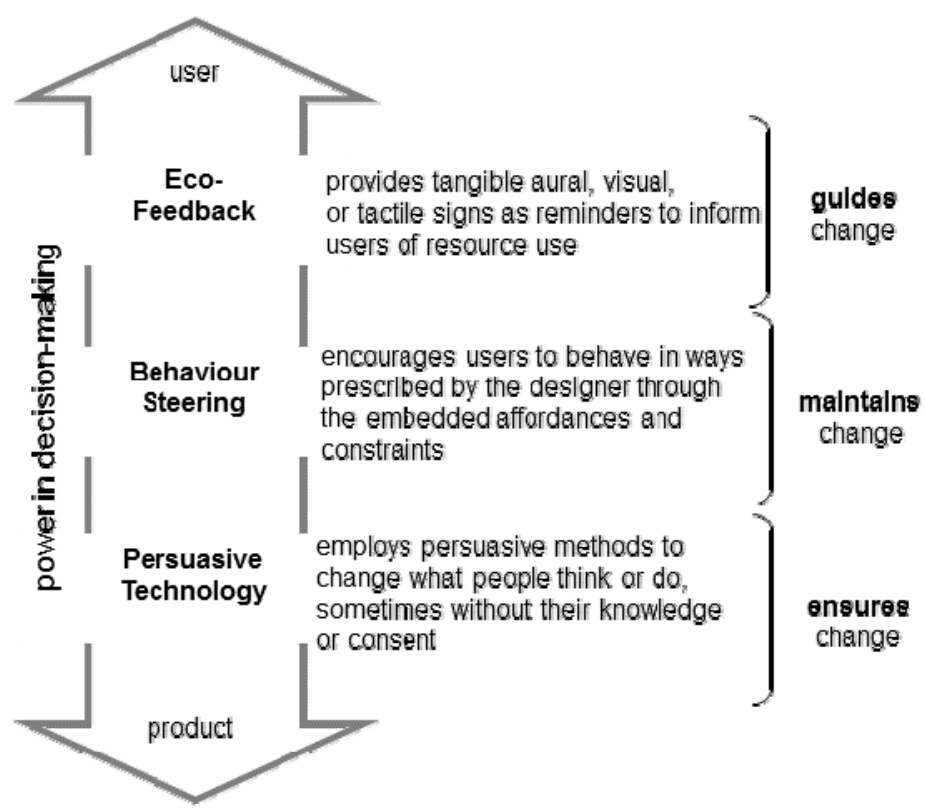

Figure 1: Strategies for designing sustainable behaviour

As seen in Figure 2, two pilot user research studies and a main user study were carried out in conjunction with a literature review to draw out perceived social impacts of mobile phone use in public. More detail on the methodology and findings of each individual study can be found in Lilley (2007).

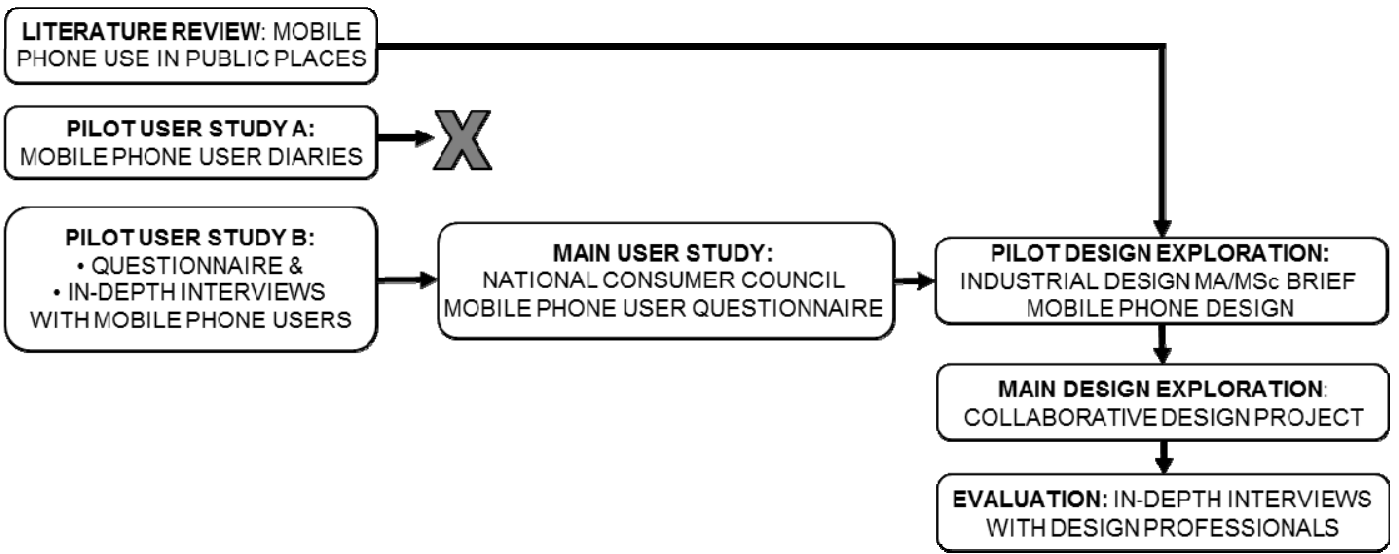

Figure 2: Case study methodology

Two design explorations followed. The pilot involved the observation and analysis of the processes and outcomes of nine Loughborough University MA/MSc Industrial Design student projects. The students were given an introductory lecture and then set a brief which challenged them to apply one of the behaviour change strategies shown in Figure 1 to the design of a mobile phone to reduce negative social impacts of its use in public. Negative behaviours were targeted for reduction as it was felt that designing out these behaviours would be of greater benefit to those affected by use. In addition to theory supporting the strategies, a range of product design case studies were provided. The students submitted a final presentation detailing their final design and a logbook illustrating how it was developed. On completion, they were interviewed collectively and surveyed individually to ascertain their perceptions of the strategies introduced.

Drawing on findings of the pilot, Lilley worked collaboratively with a designer during the main design exploration to develop a more refined response to the original brief set for the MA/MSc 
students. The resulting concept was evaluated by eight respondents from various design professions, described in Table 1, during one-to-one interviews.

Table 1: Design professionals interviewed: by type

Participant Description

Code

\begin{tabular}{|l|l|}
\hline A Freelance Eco-Fashion Designer & $\mathrm{DPI}-01$ \\
\hline A Product Design Lecturer \& owner of a small product design consultancy & $\mathrm{DPI}-02$ \\
\hline Four individuals working in a large design consultancy whose roles include; & \\
- Packaging, & $\mathrm{DPI}-03$ \\
- User Centered Research, & $\mathrm{DPI}-04$ \\
- Product Engineering, & $\mathrm{DPI}-05$ \\
- Industrial Design. & $\mathrm{DPI}-06$ \\
\hline The founder of an Eco-Design Centre working to integrate eco-design in SMEs & $\mathrm{DPI}-07$ \\
\hline $\begin{array}{l}\text { A Consultant working as part of the Business Innovation team for sustainability } \\
\text { focused NGO. }\end{array}$ & $\mathrm{DPI}-08$ \\
\hline
\end{tabular}

The responses were recorded and analysed by mapping each interviewee's perceptions on top of each other around corresponding issues relating to both the design concept and design for sustainable behaviour generally.

\section{Social Impacts of Mobile Phone Use}

Mobile phones are, by design, free of spatial restrictions, allowing the user unlimited interaction in a range of situations and spaces. The rapid assimilation of mobile phones into everyday life has modified cultural norms and practices, altering society's definition of socially acceptable behaviour within the public domain (Lasen, 2004). Despite an abundance of etiquette guides and voluntary codes of conduct, society has yet to develop any effective methods by which to deal with emergent impacts incurred from mobile phones presence. For the most part, the obligation is placed on the user to use their phone appropriately.

The findings of both the pilot and main user research studies illustrated that the use of mobile phones in public can contribute to both positive and negative societal impacts, (see Table 2). There are, of course, limitations to the data; the perceptions gathered are not representative of an entire nation and personal and societal norms are both wide-ranging and constantly shifting, therefore what is inappropriate behaviour in one person's eyes may be acceptable to someone else. However, it is clear that inappropriate, anti-social or inconsiderate mobile phone use can affect the perceived quality of life, health, well-being and happiness of others in the vicinity of use (Lilley, 2007). Reducing these impacts, therefore, is a worthy challenge for the sustainable designer, who, by definition, seeks to addresses social, as well as economic and environmental, concerns (Elkington, 1997; Bhamra and Lofthouse, 2007).

Table 2: Summary of social impacts of mobile phone use identified in UCR studies

\begin{tabular}{l} 
Social Impacts of Mobile Phone Use identified in UCR Studies \\
\hline Changing cultural expectations; \\
$9-5$ attitude to availability, instant contact, free of spatial constraints, physical space no longer \\
demarcates telephone use (fewer phone boxes). \\
\hline Changing communication practices and social norms; \\
Faster communication (letters to texts), virtual rather than physical, less pre-planning, more \\
spontaneous and informal, emergence of "texting" language. \\
\hline "Private" interactions in public space; \\
negative perceptions of individuals who use foul language and conduct inappropriate, \\
personal, extremely loud or offensive conversations within earshot, feeling angry, annoyed or \\
embarrassed when 'forced' to listen to others calls. Trade off between privacy and \\
convenience. \\
\hline Crime, Bullying \& Anti-Social Use; \\
mobile phone theft, ability to track user's locations via their mobile phone signal, the potential \\
risks of hacking associated with Bluetooth technology, "disposable" pay-as-you-go phones \\
used by criminals to communicate, terrorist use - remote detonation. \\
\hline
\end{tabular}


Body language, movement and gestures;

lack of concentration when texting or talking and walking, tendency to slow down or stop dead in the street or to block walkways, corridors or entrances, lack of concentration when driving and talking on a mobile phone, both with and without the use of a hands-free kit, using body or stance to demarcate 'private' space and to communicate intention to others, moving away from others, leaving the room, positioning oneself away from corridors or walkways, and cupping ones hand around the receiver to limit disturbance.

\section{Pilot Design Exploration: MA/MSc Industrial Design Project}

When considering the strategies, most of the students found eco-feedback easy to understand and apply but questioned its effectiveness in changing ingrained user behaviours due to the potential for the user to ignore the feedback provided. Behaviour steering was generally understood, but some students found it difficult to distinguish from persuasive technology. The application of persuasive technology took two forms; mechanistic e.g. limiting or prescribing functionality and emotional e.g. an embodied sense of self and ability to produce an emotional response to actions taken by the user. Persuasive technologies were seen as having the greatest potential for effecting change. However, some students felt that the balance of control may be weighted more heavily on the side of the product; therefore users may feel controlled or restricted. Interestingly, the majority of students did not use one approach in isolation but combined two or more approaches to increase the effectiveness and resilience of the design. The concepts generated by the design students can be seen in (Lilley et. al, 2006).

Drawing on the collective findings of the literature review carried out prior to the case study and MA/MSc project, Lilley devised an outline of promising factors, seen in Table 3, which could increase the effectiveness of "behaviour changing" devices in modifying user behaviour.

Table 3: Summary of attributes for 'Behaviour Changing' devices

\begin{tabular}{|l|l|}
\hline \multicolumn{2}{|c|}{ Summary of Attributes for 'Behaviour Changing' Devices } \\
\hline \multicolumn{2}{|c|}{ Based on the evidence, 'behaviour changing' devices should; } \\
\hline 1 & make resource use and resulting waste visible, \\
\hline 2 & be coupled with eco-efficiency improvements, \\
\hline 3 & provide tangible incentives and measurable outcomes, \\
\hline 4 & use predominately positive, rather than negative, reinforcements, \\
\hline 5 & avoid competing with other values, \\
\hline 6 & provide feedback in real-time, \\
\hline 7 & ensure reinforcements are varied in frequency and modality, \\
\hline 8 & adjust to respond to changes in user behaviour \\
\hline 9 & not compete with, but be supported by, and support, the context of use \\
\hline 10 & be, as far as possible, ethical in their intent and predicated outcomes \\
\hline
\end{tabular}

\section{$4 \quad$ Main Design Exploration: Collaborative Design Project}

The intention of this design project was to create a 'provocative' concept which could be used as a vehicle to discuss and explore the acceptability and effectiveness of potential strategies (described in Figure 1) with design professionals, as well as the wider implications of design for sustainable behaviour.

Using Caller Hegemony (Hopper, 1992) as a starting point, this concept aimed to prevent inappropriate use at the outset by encouraging users to consciously reflect on whether an interaction should take place. The designers intention was not to disregard positive impacts by focusing on reducing negative ones, but to 'design out' socially inappropriate behaviour and in doing so encourage more acceptable use.

Using sensorial inputs the mobile phone collects relevant data regarding the user behaviour and the context of use. As seen in Figure 3, It is equipped with "inherent memory" which it 
uses to determine its current state, "previous memory" which refers to learned experiences, Bluetooth connectivity, operational within a 10 metre radius, a global satellite positioning tracking system used to pinpoint the current location and to ascertain whether the phone is in transit or stationary, a 24 hour clock and voice recognition to determine whether the user is engaged in physical conversation.

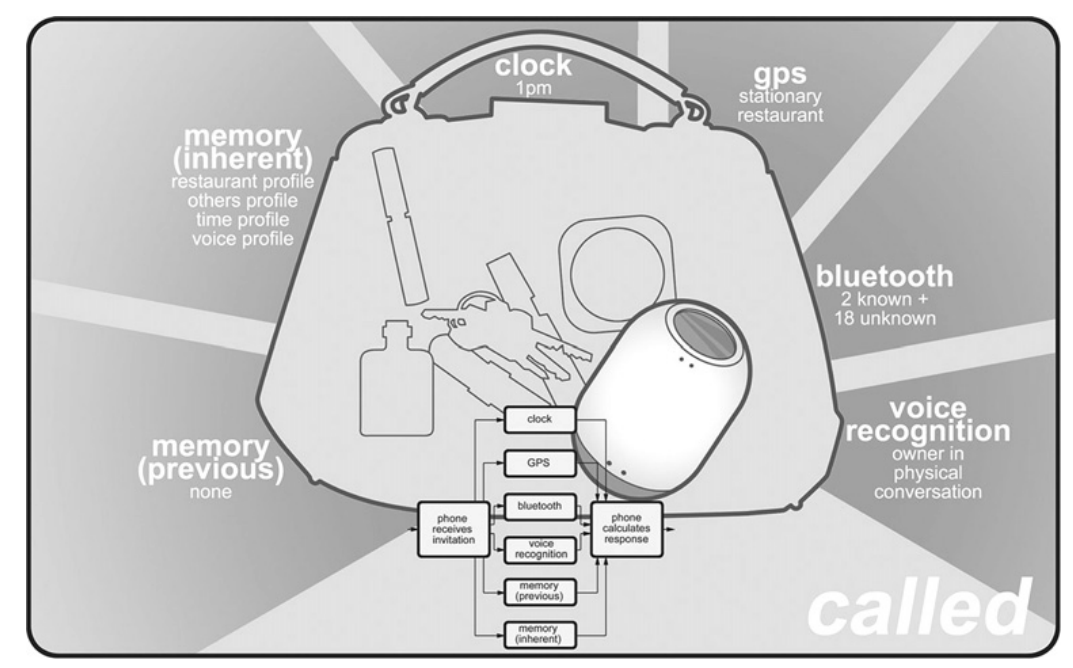

Figure 3: The mobile phone collects contextual data

Each phone is connected to a central network database ubiquitously which continuously updates itself with information provided by individual handset usage data which it uses to construct new protocols for appropriate use. The direct relationship between the phone and the network also allows for concerned users to flag up instances of anti-social behaviour. Using the "Grrr! button", the concerned user can transmit a snap shot of all phones in a 10 metre Bluetooth radius back to the network anonymously, thereby avoiding confrontation. When a particular complaint has been logged by many users the network transmits a protocol upgrade to all handsets registered on the network.

In contrast to prior design concepts identified by the Lilley (2007), this design features three levels of intervention ("passive", "assertive" and "aggressive") which are enacted in a sequential manner in response to three variables; the user's level of compliance, the gravity of the consequences of actions taken and the context in which the interaction takes place. Over time the "inherent memory" would be periodically updated with new protocols and the phone made aware of new forms of misuse via the central database but initially the phone would have a limited range of protocols to deal with three forms of misuse in a social context, drawn from prior research; "Raised Voice", "Prolonged Conversation" and the presence of "Physical Others".

\section{1 'Raised Voice' Protocol}

In response to the user raising their voice to an excessive level, the phone, in the first instance, shows it annoyance by intermittently providing aural feedback of their voice. This is a passive intervention which aims simply to remind the user of the inappropriate nature of their call. If the user continues to speak loudly it becomes more assertive and gently begins to vibrate. Continuous loud talking prompts the phone to take an aggressive stance to hinder the progress of the call by vibrating frenetically whilst increasing the aural feedback.

\section{2 'Prolonged Conversation' Protocol}

Prolonged conversations in inappropriate environments cause the phone to become increasingly bored. Analysis of a series of contributing factors determines when the call has 
exceeded a sociable duration. The phone conveys its boredom by slowing down the conversation, creating a noticeable slurring of speech. If the user persists, the level of slurring increases in an attempt to further impede the conversation and encourage the "called" to hang up. Prolonged conversation causes the battery to drain, slowing and reducing the output of dual running functions such as reading text messages whilst talking. Finally, if the user refuses to acknowledge the emotional state of the phone, and therefore their own level of anti-social behaviour, the phone begins to drain the accumulated call time by distributing the user's minutes to the phones nearby as compensation for wasting their time. Non-compliance at this stage causes the phone to eventually lapse into unconscious boredom. The phone can be reused afterwards once it regains consciousness.

\section{3 'Physical Others' Protocol}

Using the phone in an anti-social way in the presence of others causes it to display its embarrassment. It attempts to reconnect the user to the physical world by emitting a small red glow whilst randomly punctuating the conversation by switching to speakerphone mode. If the user persists in behaving inappropriately the phone becomes more embarrassed and glows deep red. This is visible to others. The frequency and the quantity of words released on speakerphone increases allowing others to partially join in the conversation. Finally the phone takes an aggressive stance by emitting a deep red glow which showers light all around the user. The speakerphone is now at full capacity preventing the use of the phone near the ear and allowing all "spectators" in the vicinity to participate in, or comment on, the conversation.

The phone retains a cumulative memory of the user's actions in the "previous memory" which it uses to compute a suitable entry point in the "passive - assertive - aggressive" scale of intervention. For example, if the user persists in shouting whilst surrounded by company in a restaurant, switching to partial speaker phone may not be a sufficient deterrent; therefore the phone may immediately default to full speakerphone.

The balance of power between the product and the user must be carefully configured to ensure trust is maintained and irritation avoided. Regular reinforcement can assist in ensuring changes in behaviour are maintained (Scott, 2004). However, repetitive reinforcement, particularly of behaviours already adopted by the user, may become annoying. Evidence suggests that varying the frequency and modality of interventions can; reduce irritation and increase spontaneity (Arroyo et al., 2005) whilst reducing the potential for users to anticipate and circumvent actions taken by the device to change their behaviour (Jelsma and Knot, 2002). Interesting and evolving interaction may also help to create and maintain the userproduct relationship. In this concept, the frequency of interventions enacted by the phone is gradually reduced as the user's compliance increases. Should the user desist at any point the phone will decline in its emotional response, thereby providing real-time feedback at the point of compliance and strengthening the link between action and consequence. By introducing a stepped process of intervention in response to variable parameters, updating "inherent memory" with evolving protocols for dealing with emergent behaviours and enabling a reciprocal exchange of educational feedback between the phone, the user, the central database and others, this concept achieves a continuous variety in both the typology and frequency of intervention.

\section{$5 \quad$ Evaluation of Collaborative Design Project}

The concept was generally well received and its presentation provoked some interesting responses which are reported on in the following sections.

\subsection{A Moral Code}

The designer's intention to introduce a "social or moral code" was recognised, but its potential for influencing user behaviour questioned. Some respondents felt that the inherent moral code would only be accepted and adhered to if it matched and reinforced the values and intentions of the user (DPI-01, DPI-04, DPI-06). Filtering out "unacceptable" or "undesirable" behaviours would be more straightforward for users with a strong moral code constructed of 
"absolutes" than those with less clearly defined guiding principles (DPI-01). It was agreed that in order to be accepted, the product would need to support, not contradict, user's values. Coinciding with a match in values was the suggestion that the product should support the user's lifestyle and tailor its interventions to suit their temperament. Assessing the "tolerance levels" of the user would be a key step in setting appropriate, personalised boundaries for interventions made (DPI-04).

\subsection{Baseline of Social Norms}

Linked to the discussion around formulating a moral code was the need for a baseline of societal norms on which to model the levels of interventions enacted as part of a protocol. This baseline would also, respondents felt, help determine the types of protocols included in the "inherent memory". Potential considerations would be how to determine the acceptable length of a conversation in a crowded restaurant or the level at which a voice becomes "too loud", for example. Keeping up with constantly changing social norms was perceived to be problematic, as norms are affected by the context of use and differing levels of acceptability. A protocol based on a projection of how people are "likely" to react may be defunct, for example, if those in the vicinity do not object to that behaviour (DPI-04).

\subsection{Relationship between Use Impact \& Strength of Intervention}

Related to the discussion of how to construct a baseline of social norms was consideration of how to rate the significance of certain behaviours in light of the severity of their impact on others. There was some discussion as to whether the use of a design intervention to limit behaviour was perhaps more justifiable if the potential consequences of such actions were severely detrimental to society. There appeared to be some consensus that product intervention may be "more acceptable when used to ensure safety" (DPI-05). A particular example being the use of mobile phones whilst driving; "you can make the case for safety in cars because it's life or death" (DPI-02). In this instance it was felt that interventions to prevent non-hands free mobile phone use in cars would be considered justifiable particularly as there is "hard data... [and] it's supported by law" (DPI-02). This in turn would increase user acceptance.

\subsection{Dominance of the Product}

The dominance of the product in determining how an interaction played out caused great unease for most respondents. The relationship between product and user has long been characterised as Master and Slave, i.e. the product's role is to serve the user; "We are used to mobile phones being in absolute subservience to us" (DPI-06). This concept, however, was seen to reverse this relationship (DPI-01). The idea that decision making could fall into the domain of the product rather than the user, even intermittently, was considered problematic. Participants questioned the acceptability and viability of deferring decision making to the product in light of the current limitations of artificial intelligence ( $\mathrm{Al}$ ). Al, they felt, would be unable to replicate complex human thought patterns and decision making, particularly in the context of mobile phone use. The system would, they felt, be "unable to represent the complexity of behaviours [and]......comprehend and analyse the meaning of data" in human terms (DPI-01). User difficulties in interfacing with the technology may also cause problems. An extremely important call may be inadvertently blocked, for example, if the phone fails to register a call as urgent. The product's inability to adequately reflect on, and respond with empathy to, the reasons behind a persistent interaction in an "inappropriate" context would cause problems. In some situations "increasingly trying to deter" the user may even be "counter-intuitive" (DPI-03). Respondents felt that inevitably these interactions would result in annoyance and frustration on the part of the "called" and that continuous frustration would result in the premature disposal of the unit.

These discussions were also interlinked with concerns over the dominant approach taken in the design of some of the protocols, namely Physical Others and Prolonged Conversation. There was an overwhelming perception, across the majority of those interviewed, that this concept used predominately negative forms of intervention to influence product use. Some of 
the features, particularly the Physical Others protocol, were singled out as examples of a perceived dominant approach, described as "controlling" (DPI-07), "confrontational" (DPI-06), "prescriptive" (DPI-05) and "more stick than carrot" (DPI-07). Although they could see merit in the concept of designing for sustainable behaviour, many interviewees struggled to find a reason why users would opt for a product which uses overtly controlling mechanisms; "the concept behind it makes sense but the application is wrong" (DPI-06). Those functions which inhibited the phones capacity to facilitate interactions, such as the Prolonged Conversation protocol, were seen as particularly counterproductive in ensuring the continued use of the product. In a general sense, interventions which raise awareness by drawing attention to a problematic behaviour were seen as more acceptable and empowering. These interventions, many felt, would encourage behaviour change without reducing the user's ability to choose how to interact with the product. The Raised Voice protocol was positioned in these terms "being made aware of having detached yourself from the people around you in a heated situation is a positive thing....likely to have a positive effect" (DPI-03); "[its] giving me the information to allow me to make a better decision" (DPI-06).

\subsection{Levels of Intervention}

The stepped nature of the interventions was generally well received. One respondent, however, suggested a need for a stage prior to "Passive" to be enacted in the user's private sphere only. This "gentle nudge" or "tap" audible or visible only to the user would "let them decide to change their behaviour before anyone else knows about it" (DPI-04), thereby avoiding embarrassment and lessening annoyance. This was reflected by another respondent who wondered if there should be a time delay to enable the phone to reprimand the user in private after the event (DPI-02). This, he felt, would be less confrontational.

\subsection{Context Awareness \& Civil Liberty}

The information gathered by sensors to inform interactions was generally seen as useful in enabling the "Caller" and the "Called" to judge the appropriateness of planned interactions. Although respondents understood the importance of gathering data to build context awareness, there were some concerns regarding the protection of civil liberties and personal privacy. This extended to include the "Caller" as well as the "Called", principally with regard to the Physical Others protocol where parts of the conversation are aired publicly. In this case in particular, participants felt that the "Caller" is at a slight disadvantage as they could be unaware of this lack of privacy. It was felt that the device should "make people at the other end aware.....that potentially their conversation is being heard" (DPI-04). To be given information regarding the "Called's" context would, some felt, infringe on their right to privacy and potentially undermine trust; particularly if used "as a tool to try to prove infidelity" (DPI-01) The resulting loss of anonymity reflected in the unbiased "honesty" of the information provided by the technology may not sit well with users who enjoy a fluid relationship with the truth when conveying their circumstances i.e. location and company. "I'm not sure people would want technology to provide that degree of honesty" "because one of things phones give you... is the ability to lie" (DPI-08). Some argued that the technology could be simpler and less invasive if the "Caller" was only given an indication of the "Called's" status i.e. busy call back later, for example (DPI-05). The crux of this debate was considered to be comprised of two points; "what information is needed to help people make better decisions" (DPI-06) and how can this information best be delivered without compromising the privacy and liberty of those affected?

\subsection{Rebound Effects}

The possibility of users' actively trying to disable or circumvent functions was considered as a potential problem for product intervention. Being humiliated, dominated or reprimanded by a product may, many felt, deter users from purchasing or continuing to use a phone with this system. It may even damage relations between consumers and manufacturers "you'd end up throwing it and vowing never to buy that brand again" (DPI-02). If product interventions caused a level of annoyance which prompted a decline in sales, manufacturers' interest in applying these approaches would arguably also decline; "you don't want people to get so annoyed with it that they're not going to use it or get the next upgrade" (DPI-04). A majority 
view was that there is a need for a manual override function which enables consumers to disable certain functions, particularly in cases of emergency; "As long as you can override it....you need to give somebody a point where they are still master of their device" (DPI-03). The problems associated with including a manual override, however, were acknowledged "if you could override it - you probably would" (DPI-04). It was suggested that it would not be inappropriate to place the onus to disable functions on the user. Users may only choose to disable automatically installed protocols if they strongly oppose their use; "I would probably argue that to work that out [how to disable functions] you'd probably have to have a desperate need to do that" (DPI-03). Therefore, it is possible to assume that default functions would, for the most part, remain active, particularly if locating the means by which to disable them was difficult or time consuming.

It was felt by some that if users purposefully try to enact interventions on their own phones or sabotage others, these phones could potentially "become tools for anti-social use" (DPI-01). Particularly by those people "who don't care less....[and] want to be the most offensive... they can be" (DPI-01). As they would "get their kicks" not by "lowering.... the impact of use but escalating it" (ibid).

\section{Discussion}

The interviews with the designers revealed an interesting perception of the trade-off between effectiveness versus acceptability. There was greater support for interventions which steer user behaviour towards more socially-conscious actions without diminishing the user's ability to choose how to interact, such as eco-feedback. The level of acceptance of eco-feedback, however, was not matched by its perceived effectiveness in prompting and sustaining changes in user behaviour. Indeed although there are plans to introduce smart -metering in homes across the UK, opinions are still divided as to whether providing informative feedback will produce the required changes in habits. Providing real-time feedback (Fogg, 2003, Arroyo et al., 2005, McCalley, 2006) and ensuring its visibility could increase the effectiveness of a device in ensuring the message is received and acted on. Its effectiveness, however, relies on an assumption that increased visibility of usage patterns, particularly relating to cost, will motivate behavioural changes. Yet information provision does not necessarily lead to action and feedback alone may not be a sufficient driver. Additionally, some consumers may need assistance to interpret feedback received and relate this information to their behaviour (Darby, 2001).

Not withstanding current technological limitations, persuasive technologies, operating ubiquitously and autonomously, have the potential to be incredibly effective, offering a more reliable and replicable method for ensuring more sustainable behaviour. The challenge associated with the use of persuasive technologies, particularly automated systems, however, is that by removing decision-making from the user and preventing 'unsustainable' actions we separate cause and effect. Without feedback on cause and effect users may be less likely to learn from, and adapt, their behaviour accordingly. They may perceive automation as a lack of choice and this may reduce acceptance. In some instances, however, users may respond positively to automation of certain actions citing convenience and time reduction as benefits. Further investigation is needed to determine where automation of actions is acceptable and where choice is preferred.

The majority of the respondents felt "uncomfortable" with the level of control exerted by persuasive technologies and the pervasive nature of their interactions, particularly "contextaware' technologies which use Bluetooth, GPS and motion sensors to gather behavioural data. The interviewee's reservations centred on the potential infringement of choice and privacy. Their concerns were particularly acute regarding mobile devices. Collecting and storing usage data on time, location and company raises questions about the security, distribution and storage of data. A persuasive technology may take advantage of personal information and use this to exert leverage to ensure the designers intention is fulfilled, it may allow information to be accessed by a third party - such as a parent, employer or spouse who may act on it to punish or reward behaviour. Additionally, unless robust security measures are integrated into the device, it may be susceptible to hacking. Therefore, "persuasive technologies that relay personal information about a user to others must be 
closely scrutinized for privacy concerns" (Berdichevsky and Neuenschwander, 1999) and safeguards put in place.

Concerns were also raised regarding individual liberty. Currently, a mobile phone user has the choice, and indeed right, to privacy with regards to their whereabouts and choice of company. Changing this dynamic would, respondents felt, change the nature of "truth" in our interactions and imbue seemingly inanimate objects with the ability to report on and "judge" user actions. Furthermore, the invasive nature of some persuasive technologies would, the respondents felt, limit their acceptance. These devices may also encourage some users to actively indulge in game playing to escalate rather than reduce their use impacts or actively seek ways to disable features to 'evade' detection, thus counteracting the designer's intentions. The introduction of an additional level of intervention prior to "passive" was a good one, as this would enable a very private exchange between product and user. The advantage of this level of intervention would be the re-introduction of a feeling of product servitude and reverence, traits currently expected by users.

A further issue raised by the respondents in response to the use of persuasive technology referred to the distribution of power between the user and the device. The overwhelming feeling of product dominance, particularly related to Physical Others and Prolonged Conversation, was interesting and unexpected. Particularly as many respondents felt even the "passive" interventions to be too intrusive. Given the current subservient nature of most products, elevating the product's level of influence to exceed that of the user and enabling autonomous actions to curb or limit user actions would almost certainly be seen as unacceptable to consumers used to being the "master" of their devices.

The majority of the respondents held different views on what an acceptable level of intervention is and what types of intervention they deemed too intrusive. This represents a challenge for the implementation of Design for Sustainable Behaviour in terms of how to classify which types of behaviour warrant more forceful interventions and what is an acceptable level of intervention. Many expressed the need for a "baseline" of social norms. The need to formulate a baseline of social norms is an interesting proposition. Its necessity is clear, particularly if there is to be coherence between product interventions and a perceived need for intervention, but it may be difficult to achieve. Keeping up with constantly changing social norms would be problematic, as norms are affected by the context of use and differing levels of acceptability. The findings indicated that it may be easier for manufacturers to justify the use of more forceful interventions if the target behaviours are already legislated against, widely deemed as socially unacceptable, behaviours which diminish or challenge personal or public safety or are illegal. These interventions would, arguably, also be more readily accepted by users as legitimate concerns, and therefore meet less resistance. Reaching a consensus on how to rate the severity of consequences enacted by different behaviours, and deciding on an appropriate level of intervention, would, they felt, be challenging as decisions would almost certainly be subjective. Additionally, there is the question of who would lead such an ambitious undertaking. It is possible to speculate that most manufacturers may not be entirely comfortable rating the potentially negative consequences of the use of their product.

\section{Conclusions}

There is not, as yet, a clear consensus of what is an acceptable level of intervention, or how to rate the severity of consequences enacted by different behaviours. The consequences of use differ from one product to another, therefore, to enable manufacturers to identify and rate product specific impacts and put measures in place in the product design to prevent potential or foreseeable consequences resulting from use, they must observe how people use and misuse existing products.

The hypothesises of this research is that a range of interventions from "passive" (or "informative") to "assertive" (or "persuasive") to "aggressive" (or "coercive") should be enacted in a sequential manner in response to three variables;

- the user's level of compliance,

- the gravity of the consequences of actions taken, 
- the context in which the interaction takes place.

Where to place each approach on this continuum, however, is open to discussion. To accurately classify interventions on this scale more research gathering user perceptions to measure their tolerance levels is needed. Additionally, further investigation is needed to determine where automation of actions is acceptable and where choice is preferred.

The intentionality of the designer is an important concern as this, coupled an assessment of the severity of the consequences of product use or misuse, will inform the selection of a strategy. The intended behavioural outcomes of the intervention must be clear, well defined and justifiable. In addition, more research is needed to understand the ethical dimensions of applying behaviour change strategies and how to identify and assess these factors. Finding an acceptable level of product influence through intervention and ensuring the moral acceptability of such interventions will be key to ensuring consumer acceptance and manufacturer buy-in.

Designers and engineers can positively influence product use if decisions are made at a strategic level prior to design development. In order to implement a DfSB strategy, however, manufacturers must either voluntarily accept some responsibility for product impacts beyond point-of-purchase or have a compelling reason to do so. Unless they could see a marketable advantage, respondents did not believe that most manufacturers would voluntarily introduce strategies for influencing user behaviour to reduce use impacts unless the public's negative perceptions of the social or environmental impacts product use reached a critical mass or 'tipping point' resulting in adverse publicity or unless legislation was introduced. The justification for product intervention may be more readily accepted, and meet with less resistance, if the target behaviours are already legislated against, widely deemed as socially unacceptable, behaviours which diminish or challenge personal or public safety or are illegal. Classifying what is, and what isn't, socially acceptable behaviour may prove challenging, however, as social norms are constantly evolving.

Those who do opt to modify user behaviour would do so cautiously whilst taking into account other business factors, notably economic growth and profit. Coercive interventions may prove more successful in altering behaviour, but consumer acceptance of these devices is most likely to be low and therefore manufacturers' willingness to adopt these approaches limited. After all most manufacturers prime concern is to make money. In the case of mobile phones, a market where profit depends on the increased usage of communication services, advocating talking less would be commercial suicide. Any design intervention in this industry would need to emphasise communicating differently not less. In short, responsible use must not mean reduced use.

\section{Acknowledgements}

Many thanks to Dr Tracy Bhamra, Dr Vicky Lofthouse, Garrath T Wilson and all the design professionals who generously gave their time, opinions and expertise.

\section{Figure Captions}

Figure 1: Strategies for Designing Sustainable Behaviour

Figure 2: Case Study Methodology

Figure 3: The Mobile Phone Collects Contextual Data

\section{Table Captions}

Table 1: Design Professionals Interviewed: by Type

Table 2: Summary of Social Impacts of Mobile Phone Use identified in UCR Studies

Table 3: Summary of Attributes for 'Behaviour Changing' Devices

\section{References}

Akrich, M. (1992) The De-Scription of Technical Objects In: Shaping Technology: Building Society, Bijker, W. E. and Law, J. (eds) Massachusetts Institute of Technology (MIT), Cambridge, Massachusetts, pp. 205-224. 
Arroyo, E., Bonanni, L. and Selker, T. (2005) Waterbot: Exploring Feedback and Persuasive Techniques at the Sink In: $\mathrm{CHI}$ 2005, Portland, Oregon, USA 2nd - 7th April 2005

Baines, J. and Morgan, B. (2004) Sustainability Appraisal: A Social Perspective In: Sustainability Appraisal: A Review of International Experience and Practice, Datal-Clayton, B. and Sadler, B. (eds) First Draft of Work in Progress, International Institute for Environment and Development, London.

Berdichevsky, D. and Neuenschwander, E. (1999) Towards an Ethics of Persuasive Technology, Communications of the ACM, Vol. 42, No. 5, pp. 51-58.

Bhamra, T. and Lofthouse, V. (2007) Design for Sustainability: A Practical Approach, Gower, UK.

Bhamra, T. A., Lilley. D and Tang, T. (2008) Sustainable Use: Changing consumer behaviour through product design, In: Changing the Change: Design visions, proposals and tools, Turin, Italy, 10th-12th July 2008

Colantonio, A. (2007) Social Sustainability: An Exploratory Analysis of its Definition, Assessment Methods, Metrics and Tools, Oxford Institute for Sustainable Development, 2007/01: EIBURS Working Paper Series, July 2007.

Darby, S. (2001) Making it obvious: designing feedback into energy consumption In: 2nd International Conference on Energy Efficiency in Household Appliances and Lighting, Italian Association of Energy Economists/ EC-SAVE programme

DeVries, M. J. (2006) Ethics and the complexity of technology: a design approach, Philosophia Reformata, the international scientific journal of the Association for Reformational Philosophy, Vol. 71, No. 2, pp. 118-131.

Elias, E. W. A., Dekoninck, E. A. and Culley, S. J. C. (2008) Assessing User Behaviour for Changes in the Design of Energy Using Domestic Products In: IEEE International Symposium on Electronics and the Environment (ISEE), San Francisco, California, US, 19th - 22nd May

Elkington, J. (1997) Cannibals with forks: the triple bottom line of 21st century business, Capstone, Oxford.

Fogg, B. J. (2003) Persuasive Technology; Using Computers to Change What We Think and Do, Morgan Kaufmann, San Fransisco.

Hopper, R. (1992) Telephone Conversation, Indiana University Press, Bloomington, Indianapolis.

Jelsma, J. and Knot, M. (2002) Designing environmentally efficient services; a 'script' approach, The Journal of Sustainable Product Design, Vol. 2, pp 119-130.

Lewis, H., Gertsakis, J., Grant, T., Morelli, N. and Sweatman, A. (2001) design + environment, Greenleaf, Sheffield, UK.

Lilley. D (2007) Designing for Behavioural Change: Reducing the Social Impacts of Product Use through Design, Doctoral Thesis, Department of Design and Technology, Loughborough University

Lilley. D, Bhamra T.A and Lofthouse. V. A (2006) Towards Sustainable Use: An Exploration of Designing for Behavioural Change In: Design and Semantics of Form and Movement DeSForM 2006 Evoluon, Eindhoven 26th - 27th October 2006

Lasen, A. (2004) Affective technologies - emotion and mobile phones, Reciever, 11, Vodaphone.

Lockton, D., Harrison, D. and Stanton, N. (2008) Making the user more efficient: Design for sustainable behaviour, International Journal of Sustainable Engineering, Vol. 1, No. 1, pp. 3-8.

McCalley, L. T. (2006) From motivation and cognition theories to everyday applications and back again: the case of product-integrated information and feedback, Energy Policy, Vol. 34, No. 2, pp. 129-137.

McCalley, L. T. and Midden, C. J. H. (2006) Making Energy Feedback Work In: User Behavior and Technology Development: Shaping Sustainable Relations Between Consumers and Technologies Verbeek, P.-P. and Slob, A. (eds) Springer, The Netherlands, pp. 127-135.

Papanek, V. (1971) Design for the Real World: Human Ecology and Social Change, Pantheon Books, New York. 
Polese, M. and Stren, R. (2000) The Social Sustainability of Cities: Diversity and the Management of Change, University of Toronto Press, Toronto.

Scott, F. (2004) Behaviour Change - believing you can make a difference! In: BTCV, Global Action Plan and The Environment Council Workshop, London 20th July 2004

Siegle, L. (2006) Your home may be hurting the planet, Guardian Unlimited, Thursday 8th June 2006.

Sinner, J., Baines, J., Crengle, H., Salmon, G., Fenemor, A. and Tipa, G. (2004) Sustainable Development: A Summary of Key Concepts, Ecologic Research, Report No. 2.

Wever, R., van Kuijk, J. and Boks, C. (2008) User-centred Design for Sustainable Behaviour International Journal of Sustainable Engineering, Vol. 1, No. 1, pp.9-20. 